\title{
Assessment and management of bipolar disorder: summary of updated NICE guidance
}

\author{
Tim Kendall director, consultant psychiatrist ${ }^{12}$, Richard Morriss professor of psychiatry and community \\ mental health ${ }^{3}$, Evan Mayo-Wilson assistant scientist ${ }^{4}$, Elena Marcus research assistant ${ }^{56}$, on \\ behalf of the Guideline Development Group
}

\begin{abstract}
${ }^{1}$ National Collaborating Centre for Mental Health, Royal College of Psychiatrists, London E1 8AA, UK; ${ }^{2}$ Sheffield Health and Social Care NHS Foundation Trust, Sheffield S10 3TH, UK; ${ }^{3}$ Psychiatry and Applied Psychology, Faculty of Medicine and Health Sciences, University of Nottingham, Nottingham, UK; ${ }^{4}$ Department of Epidemiology, Johns Hopkins Bloomberg School of Public Health, Baltimore, MD, USA; ${ }^{5}$ National Collaborating Centre for Mental Health, University College London, London, UK; ${ }^{6}$ Centre for Outcomes Research and Effectiveness, University College London, London, UK
\end{abstract}

This is one of a series of $B M J$ summaries of new guidelines based on the best available evidence; they highlight important recommendations for clinical practice, especially where uncertainty or controversy exists.

Bipolar disorder is a complex, recurrent, and severe mental illness that has an onset typically between 13 and 30 years of age and a lifetime prevalence of $1.4 \%{ }^{1}$ It is characterised by episodes of mania or hypomania with elation, overactivity, and disinhibited behaviour, as well as episodes of depression with profound loss of interest and motivation, often with milder depressed mood in between episodes. Bipolar disorder is associated with an increased risk of suicide and physical illness, such as ischaemic heart disease, diabetes, chronic obstructive airways disease, pneumonia, and unintentional injury. ${ }^{2}$ Around two thirds of people with bipolar disorder also experience another mental disorder, usually anxiety disorders, substance misuse disorders, or impulse control disorders. ${ }^{1}$ The risk of recurrence in the year after a mood episode is especially high (50\% in one year and $>70 \%$ at four years) compared with other psychiatric disorders, ${ }^{3}$ and this has important implications for the long term management of the disorder.

This article summarises the most recent recommendations from the National Institute for Health and Care Excellence (NICE) on assessing and managing bipolar disorder in adults, children, and young people. ${ }^{4}$

\section{Recommendations}

NICE recommendations are based on systematic reviews of the best available evidence and explicit consideration of cost effectiveness. When minimal evidence is available, recommendations are based on the Guideline Development Group's experience and opinion of what constitutes good practice. Evidence levels for the recommendations are given in italic in square brackets.

\section{Care for adults, children, and young people across all phases of bipolar disorder}

- Use this guideline in conjunction with the NICE clinical guidance on service user experience in adult mental health ${ }^{5}$ to improve the experience of care by:

-Promoting a positive recovery message from the point of diagnosis and throughout care

-Building supportive and empathic relationships as an essential part of care. (New recommendation.) [Based on the experience and opinion of the Guideline Development Group $(G D G)$ and on evidence reviewed in one recent NICE guideline on mental health (CG136) ]

- As early as possible negotiate with the person with bipolar disorder and his or her carers about how information about the person will be shared. When discussing rights to confidentiality, emphasise the importance of sharing information about risks and the need for carers to understand the person's perspective. Foster a collaborative approach that supports people with bipolar disorder and their carers and also respects their individual needs and interdependence. (New recommendation.) [Based on the experience and opinion of the GDG and on evidence reviewed in one recent NICE guideline on mental health (CG178) ]

- When using any psychotropic drugs for bipolar disorder ensure that: 
-People are given information that is suitable for their developmental level about the purpose and likely side effects of treatment, including any monitoring that is needed, and give them an opportunity to ask questions

-Drugs are chosen in collaboration with the person with bipolar disorder, taking into account the carer's views if the person agrees

-The overall medication regimen is regularly reviewed so that drugs that are not needed after the acute episode are stopped. (New recommendation.) [Based on the experience and opinion of the GDG]

\section{Recognising and managing bipolar disorder in adults in primary care}

- When adults present in primary care with depression, ask about previous periods of overactivity or disinhibited behaviour. If the overactivity or disinhibited behaviour has lasted for four days or more, consider referral for a specialist mental health assessment. Refer people urgently for a specialist mental health assessment if mania or severe depression is suspected (see box for key symptoms or refer to the ICD-10 (international classification of diseases, 10th revision) for full diagnostic criteria ${ }^{7}$ ) or they are a danger to themselves or others. (New recommendation.) [Based on the experience and opinion of the $G D G]$

- Monitor the physical health of people with bipolar disorder when responsibility for monitoring is transferred from secondary care, and then at least annually. The health check should be comprehensive and focus on physical health problems, such as cardiovascular disease, diabetes, obesity, and respiratory disease. A copy of the results should be sent to the care coordinator and psychiatrist, and it should be put in the secondary care records. (New recommendation.) [Based on the experience and opinion of the GDG and on evidence reviewed in one recent NICE guideline on mental health (CG178)] ${ }^{6}$

- Offer people with rapid cycling bipolar disorder the same interventions as people with other types of bipolar disorder because there is currently no strong evidence to suggest that people with rapid cycling bipolar disorder should be treated differently. (New recommendation.) [Based on the experience and opinion of the GDG]

\section{Managing mania or hypomania in adults in secondary care}

- If a person develops mania or hypomania and is not taking an antipsychotic or mood stabiliser, offer haloperidol, olanzapine, quetiapine, or risperidone, taking into account any advance statements, the person's preference, and clinical context (including physical comorbidity, previous response to treatment, and side effects). If the person is already taking lithium, check plasma lithium levels to optimise treatment and consider adding haloperidol, olanzapine, quetiapine, or risperidone. Do not offer lamotrigine to treat mania. (New recommendation.) [Based on a systematic review and network meta-analysis, ${ }^{8}$ and on the experience and opinion of the $G D G]$

- If a person develops mania or hypomania and is taking an antidepressant (as defined by the $B N F^{9}$ ) as monotherapy: -Consider stopping the antidepressant and
-Offer an antipsychotic regardless of whether the antidepressant is stopped. (New recommendation.) [Based on the experience and opinion of the GDG]

\section{Managing bipolar depression in adults in secondary care}

- Offer adults with bipolar depression:

-A psychological intervention that has been developed specifically for bipolar disorder and has a published evidence based manual describing how it should be delivered or

-A high intensity psychological intervention (cognitive behavioural therapy, interpersonal therapy, or behavioural couples therapy) in line with the NICE clinical guideline on depression. ${ }^{10}$

Discuss the possible benefits and risks of psychological interventions and the person's preference. Monitor mood for signs of mania or hypomania or deterioration of the depressive symptoms. (New recommendation.) [Based on a systematic review and meta-analysis, and on evidence reviewed in one recent NICE guideline on mental health (CG90) $]^{10}$

- If a person develops moderate or severe bipolar depression and is not taking a drug to treat the disorder, offer fluoxetine combined with olanzapine, or quetiapine on its own, depending on the person's preference and previous response to treatment.

- If the person prefers, consider either olanzapine (without fluoxetine) or lamotrigine on its own.

- Consider the use of lamotrigine if there is no response to fluoxetine combined with olanzapine or to quetiapine on its own. (New recommendation.) [Based on a systematic review, network meta-analysis, and economic model]

- If a person develops moderate or severe bipolar depression and is already taking lithium, check the person's plasma lithium level. If it is inadequate, increase the dose of lithium; if it is at maximum level, add either fluoxetine combined with olanzapine or quetiapine on its own, depending on the person's preference and previous response to treatment.

- If the person prefers, consider adding olanzapine (without fluoxetine) or lamotrigine to lithium.

- If there is no response to the addition of fluoxetine combined with olanzapine or quetiapine on its own, stop the additional treatment and consider adding lamotrigine to lithium. (New recommendation.) [Based on a systematic review, network meta-analysis and economic model, and the experience and opinion of the GDG]

\section{Managing bipolar disorder in adults in the longer term in secondary care}

- After each episode of mania or bipolar depression, discuss the longer term management of the disorder with the person and the carers, if appropriate. Discussion should aim to help people understand that bipolar disorder is often a long term relapsing and remitting condition that needs self management and engagement with primary and secondary care professionals and the involvement of carers. The discussion should cover:

-The nature and variable course of bipolar disorder

-The role of psychological and pharmacological interventions to prevent relapse and reduce symptoms 


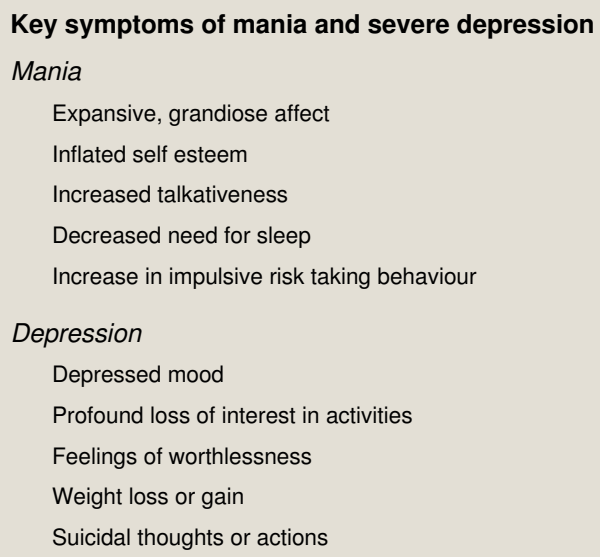

(1)


continue antipsychotic treatment for longer than 12 weeks. (New recommendation.) [Based on NICE technology appraisal $292^{12}$ and the experience and opinion of the $G D G]$

- Do not offer valproate to girls or young women of childbearing potential. (New recommendation.) [Based on the experience and opinion of the GDG]

- Offer a structured psychological intervention (individual cognitive behavioural therapy or interpersonal therapy) to young people with bipolar depression. The intervention should last for at least three months and have a published evidence based manual that describes how it should be delivered. (New recommendation.) [Based on the experience and opinion of the GDG and the content of other recommendations in the guideline]

\section{Overcoming barriers}

There is a perception that because bipolar disorder has important genetic and biological influences it must be treated with drugs, and the drug of choice for bipolar depression for many professionals is an antidepressant. ${ }^{14}$ This misconception is challenged by this guideline, which finds that psychological treatments are effective in the treatment of bipolar depression in primary and secondary care and in preventing recurrence. Psychological treatments are also important for young people, both because young people may benefit from them and because they are at greater risk of harm with drug treatment ${ }^{15}$ However access to psychological therapies is currently insufficient for adults and young people with bipolar disorder. For adults, this guideline also finds that some antipsychotics are effective in the treatment of bipolar depression, but lithium (rather than antipsychotics) should be the first line drug to prevent relapse. A major driver for these changes will be the establishment of quality networks and national audit. In adults, many mental health workers have taken the view that smoking, not taking exercise, and having a poor diet are unimportant compared with the enormous impact of a bipolar illness. ${ }^{16}$ These beliefs have contributed to people with bipolar disorder dying from preventable diseases much earlier than others. ${ }^{2}$ This guideline emphasises the importance of maintaining good physical health for people with severe mental health problems, such as bipolar disorder, and we hope it will contribute to a change in professional attitudes and practice.

There has been much controversy over the diagnosis and treatment of children and young people with possible bipolar disorder. In the United States, children as young as 4 or 5 years are being treated with drugs, ${ }^{17}$ using criteria for diagnosis that would not be accepted in the NHS. Thus, increasing numbers of children and young people are taking antipsychotics, ${ }^{18}$ which are associated with serious adverse events, including increased prolactin levels, rapid weight gain, and increased risk of diabetes. ${ }^{12}$ The previous NICE guideline rejected the extension of bipolar disorder into childhood and suggested that clinicians apply stricter criteria to adolescents, ${ }^{19}$ but the diagnosis and treatment of children and adolescents has continued, mostly in the US. ${ }^{18}$ This is despite evidence that symptoms are not stable over time ${ }^{20}$ and despite limited evidence that young people benefit from the treatments commonly used in adults. Importantly, trials in the US show that many children under 13 years diagnosed as having bipolar disorder also have attention-deficit/hyperactivity disorder, ${ }^{17}{ }^{21}$ which might be a more appropriate diagnosis. The updated guideline reinforces the findings of the last guideline and finds that extensive use of this diagnosis in children could cause more harm than good.
The members of the Guideline Development Group were Richard Morriss (chair), Tim Kendall (facilitator), Evan Mayo-Wilson, Ifigeneia Mavranezouli, Ruth Braidwood, Richard Byng, Andrea Cipriani, Korin Garcia-Niño, John Geddes, Kate Hughes, Anthony James, Carnice John, Steven Jones, Katherine Leggett, Elena Marcus, Tim McDougall, Thomas Meyer, Carol Paton, Matthias Schwannauer, Sarah Stockton, Donna Swinden, Clare Taylor, Robert Westhead, Craig Whittington, and Faye Wilson.

Contributors: All authors contributed to the conception and drafting of this article and revised it critically. They have all approved this version. TK is guarantor.

Competing interests: We have read and understood BMJ policy on declaration of interests and declare the following interests: TK, EM-W, and EM had support from the National Collaborating Centre for Mental Health, which was in receipt of funding from NICE, for the submitted work; RM received support from the National Institute for Health Research Collaboration for Leadership in Applied Health Research and Care East Midlands. The authors' full statements can be viewed at: www.bmj.com/content/bmj/349/bmj.g5673/related\#datasupp.

Provenance and peer review: Commissioned; not externally peer reviewed.

1 Merikangas KR, Akiskal HS, Angst J, Greenberg PE, Hirschfeld RM, Petukhova M, et al. Lifetime and 12-month prevalence of bipolar spectrum disorder in the national comorbidity survey replication. Arch Gen Psychiatry 2007;64:543-52

2 Crump C, Sundquist K, Winkleby MA, Sundquist J. Comorbidities and mortality in bipolar disorder: a Swedish national cohort study. JAMA Psychiatry 2013;70:931-9.

3 Harrow M, Goldberg JF, Grossman LS, Meltzer HY. Outcome in manic disorders. A naturalistic follow-up study. Arch Gen Psychiatry 1990;47:665-71.

$4 \quad$ National Institute for Health and Clinical Excellence. Bipolar disorder (update): the management of bipolar disorder in adults, children and adolescents in primary and secondary care. (Clinical Guideline 185.) 2014. http://guidance.nice.org.uk/CG185.

5 National Institute for Health and Clinical Excellence. Service user experience in adult mental health: improving the experience of care for people using adult NHS mental health services. (Clinical Guideline 136.) 2011. http://guidance.nice.org.uk/CG136.

6 National Institute for Health and Care Excellence. Psychosis and schizophrenia in adults: treatment and management. (Clinical Guideline 178). 2014. http://guidance.nice.org.uk/ CG178.

7 WHO. The ICD-10 classification of mental and behavioural disorders: clinical descriptions and diagnostic guidelines. 1992.

8 Cipriani A, Barbui C, Salanti G, Rendell J, Brown R, Stockton S, et al. Comparative efficacy and acceptability of antimanic drugs in acute mania: a multiple-treatments meta-analysis. Lancet 2011;378:1306-15.

9 Joint Formulary Committee. BNF. BMJ Group and Pharmaceutical Press. 2013. http:// www.bnf.org/bnf/index.htm.

10 National Institute for Health and Clinical Excellence. Depression in adults: the treatment and management of depression in adults. (Clinical Guideline 90.) 2009. http://guidance. nice.org.uk/CG90.

11 National Institute for Health and Care Excellence. Bipolar disorder (update): the management of bipolar disorder in adults, children and adolescents in primary and secondary care. (Clinical Guideline 185.) 2014. www.nice.org.uk/guidance/CG185/ InformationForPublic.

12 National Institute for Health and Care Excellence. Aripiprazole for treating moderate to severe manic episodes in adolescents with bipolar I disorder. (Technology Appraisal 292.) 2013. http://publications.nice.org.uk/aripiprazole-for-treating-moderate-to-severe-manicepisodes-in-adolescents-with-bipolar-i-disorder-ta292.

13 Paediatric Formulary Committee. BNF for children. BMJ Group, Pharmaceutical Press, and RCPCH Publications. 2013. www.bnf.org/bnf/org_450055.htm.

14 Blanco C, Laje G, Olfson M, Marcus SC, Pincus HA. Trends in the treatment of bipolar disorder by outpatient psychiatrists. Am J Psychiatry 2002;159:1005-10.

15 Jureidini JN, Doecke CJ, Mansfield PR, Haby MM, Menkes DB, Tonkin AL. Efficacy and safety of antidepressants for children and adolescents. BMJ 2004;328:879-83.

16 Howard L, Gamble C. Supporting mental health nurses to address the physical health needs of people with serious mental illness in acute inpatient care settings. J Psychiatr Ment Health Nurs 2011;18:105-12.

17 Findling RL, Youngstrom EA, McNamara NK, Stansbrey RJ, Wynbrandt JL, Adegbite C, et al. Double-blind, randomized, placebo-controlled long-term maintenance study of aripiprazole in children with bipolar disorder. J Clin Psychiatry 2012;73:57-63.

18 Olfson M, Crystal S, Huang C, Gerhard T. Trends in antipsychotic drug use by very young, privately insured children. J Am Acad Child Adolesc Psychiatry 2010;49:13-23.

19 National Institute for Health and Clinical Excellence. Bipolar disorder: the management of bipolar disorder in adults, children and adolescents in primary and secondary care. (Clinical Guideline 38.) 2006. http://guidance.nice.org.uk/CG38.

20 Tijssen MJ, van Os J, Wittchen HU, Lieb R, Beesdo K, Mengelers R, et al. Prediction of transition from common adolescent bipolar experiences to bipolar disorder: 10-year study. Br J Psychiatry 2010;196:102-8.

21 Wagner KD, Redden L, Kowatch RA, Wilens TE, Segal S, Chang K, et al. A double-blind, randomized, placebo-controlled trial of divalproex extended-release in the treatment of bipolar disorder in children and adolescents. J Am Acad Child Adolesc Psychiatry 2009;48:519-32. 


\section{Further information on the guidance}

An important prompt for this guideline update was a high quality network meta-analysis of treatments for acute mania published in 2011, in which haloperidol, olanzapine, quetiapine, and risperidone emerged as the most effective treatments. ${ }^{8}$ The previous guideline recommended a range of other alternatives alongside antipsychotics, including valproate and lithium, interventions which were ranked much lower in the network.

\section{Methods}

This guideline, which is an update of NICE clinical guideline $38,{ }^{19}$ was developed by the National Collaborating Centre for Mental Health using NICE guideline methods (www.nice.org.uk/guidelinesmanual). The guideline review process involved comprehensive and systematic literature searches to identify relevant evidence for the clinical and economic reviews, with critical appraisal of the quality of the identified evidence. Results of intervention studies were compared using pair-wise and network meta-analyses. Results of the network meta-analysis were used to inform an economic model. A multidisciplinary team of healthcare professionals from psychiatry, psychology, pharmacology, occupational therapy, general practice, and nursing, as well as service user and carer representatives (forming the GDG) was established to review the evidence and develop the subsequent recommendations. The guideline then went through an external consultation with stakeholders. The GDG considered the stakeholders' comments, reanalysed the data where necessary, and modified the guideline as appropriate.

NICE has produced three different versions of the guideline: a full version; a summary version known as the "NICE guideline;" and a version for children, young people, and adults with bipolar disorder, their parents and carers, and the public. ${ }^{11}$ All these versions, as well as a pathway, are available from the NICE website. Further updates of the guideline will be produced as part of NICE's guideline development programme.

Future research

-What is the clinical and cost effectiveness of structured psychological interventions for young people with bipolar depression?

- In the maintenance treatment of bipolar disorder, what are the relative effects on quality of life of lithium, an antipsychotic (haloperidol, olanzapine, quetiapine or risperidone), or a combination of lithium and an antipsychotic?

- What is the clinical and cost effectiveness of fluoxetine combined with olanzapine versus an alternative selective serotonin reuptake inhibitor combined with olanzapine in the treatment of moderate to severe bipolar depression?

- What is the clinical and cost effectiveness of a specialised collaborative care service for people admitted to hospital with bipolar disorder compared with usual treatment delivered by generic care services?

- What is the clinical and cost effectiveness of face to face cognitive behavioural therapy compared with internet facilitated cognitive behavioural therapy in the long term management of bipolar disorder? 